\title{
How much insulin resistance in polycystic ovary syndrome? Comparison of HOMA-IR and insulin resistance (Belfiore) index models
}

\author{
Krzysztof C. Lewandowski ${ }^{1,2}$, Elżbieta Skowrońska-Jóźwiak ${ }^{1,2}$, Katarzyna Łukasiak ${ }^{1}$, \\ Katarzyna Gałuszko ${ }^{1}$, Aleksandra Dukowicz ${ }^{1}$, Magdalena Cedro ${ }^{1}$, Andrzej Lewiński ${ }^{1,2}$
}

\author{
${ }^{1}$ Department of Endocrinology and Metabolic Diseases, Medical University of Lodz, \\ Lodz, Poland \\ ${ }^{2}$ Department of Endocrinology and Metabolic Diseases, Polish Mother's Memorial \\ Hospital - Research Institute, Lodz, Poland
}

Submitted: 5 December 2016

Accepted: 5 April 2017

Arch Med Sci 2019; 15 (3): 613-618

DOI: https://doi.org/10.5114/aoms.2019.82672

Copyright $@ 2019$ Termedia \& Banach

\section{Abstract}

Introduction: Polycystic ovary syndrome (PCOS), the commonest endocrinopathy of women in reproductive age, is often accompanied by insulin resistance (IR), hirsutism and/or fertility problems. The aim of the study was to assess the prevalence of IR in women diagnosed with PCOS.

Material and methods: The study involved 137 women diagnosed with PCOS, according to the Rotterdam consensus criteria (2003). Insulin resistance was assessed according to the HOMA-IR method and insulin resistance (Belfiore) index (IRI) derived from glucose and insulin during the oral glucose tolerance test.

Results: There was a significant $(p<0.0001)$ but relatively moderate correlation between IRI and HOMA-IR ( $r=0.5$ and $r=0.57$ for a linear and non-linear model, respectively). Insulin resistance was more prevalent according to IRI (49.6\%) than according to HOMA-IR $(22.6 \%$ and $15.8 \%$ for 3.46 and 3.8 cut-off points, respectively, $p<0.01)$. The majority of patients with high HOMA-IR also had high IRI (e.g. 86\%, for HOMA > 3.8), but the majority of patients with raised IRI would not be diagnosed as insulin resistant according to HOMA (61.7\% and $73.5 \%$, for HOMA-IR ${ }_{3.46}$ and $\mathrm{HOMA}-\mathrm{IR}_{3.80}$, respectively).

Conclusions: The insulin resistance (Belfiore) index indicates more cases of insulin resistance than HOMA-IR in women with PCOS. Therefore, detection of insulin resistance among women with PCOS is highly method-dependent with more severe cases being detected with HOMA-IR than with IRI.

Key words: insulin resistance, polycystic ovary syndrome, HOMA, Belfiore index.

\section{Introduction}

The term polycystic ovarian syndrome (PCOS) represents a heterogeneous and multifaceted entity characterised by hyperandrogenism and/ or ovulatory dysfunction. It is also the most common endocrinopathy of women of reproductive age [1], and is associated with an increased cardiovascular risk [2, 3]. According to the Rotterdam criteria (2003) [4] a diagnosis of PCOS can be established when at least two out of three criteria are present (oligo-/anovulation, clinical hyperandrogenism or biochemical hyperandrogenaemia and polycystic ovaries) on condition that other causes of oligo-/anovulation or hyperandrogenism/hyper-

\author{
Corresponding author: \\ Prof. Andrzej Lewinski MD, PhD \\ Department of \\ Endocrinology and \\ Metabolic Diseases \\ Medical University \\ of Lodz \\ 281/289 Rzgowska St \\ 93-338 Lodz, Poland \\ Phone: +48 422711142 \\ Fax: +48 42 2711140 \\ E-mail: alewin@csk.umed. \\ lodz.pl
}


androgenaemia (hyperprolactinaemia, Cushing's syndrome, congenital adrenal hyperplasia, premature ovarian failure, hypothalamic/pituitary disease, etc.), have been ruled out. It is well recognized that a significant proportion of women with PCOS have a propensity towards obesity, dyslipidaemia, insulin resistance, as well as abnormal glucose homeostasis (impaired fasting glucose, impaired glucose tolerance, type 2 diabetes) $[5,6]$.

Several mechanisms have been implicated in the pathogenesis of insulin resistance in PCOS, including abnormalities in pituitary gonadotropin secretion [7], excessive stimulation of IGF-I receptors, excessive activity of $17 \alpha$-hydroxylase, an enzyme that regulates conversion of 17-hydroxy-progesterone into androstenedione, as well as diminished synthesis of insulin-like growth factor binding protein 1 (IGF-BP1) [8, 9].

Though some authors claim that insulin resistance may be found in up to $70 \%$ of women with PCOS [10], while in a study of Legro et al. [11], based on the data from 254 patients, impaired glucose tolerance was found in $31 \%$ of obese and $10.3 \%$ of lean women with PCOS, the genuine prevalence of these abnormalities is indeed highly dependent both on the characteristics of the studied population and its ethnic profile [12]. There is however universal agreement that all women with PCOS should be assessed for abnormalities typical for the metabolic syndrome, including lipids and oral glucose tolerance test [13].

Though euglycaemic hyperinsulinaemic clamp technique is considered the gold standard in the assessment of insulin resistance [14], this method is too cumbersome for standard clinical practice. Hence, several other methods have been designed including a homeostatic (HOMA) model based on fasting glucose and fasting insulin (HOMA-IR $=$ [glucose $](\mathrm{mmol} / \mathrm{l}) \times$ insulin $](\mu \mathrm{U} / \mathrm{ml}) / 22.5)$ [15], or a QUICKI index (QUICKI $=1 /[\log (I(0))+$ $\log (G(0))]$, where $I(0)$ denotes fasting insulin, and glucose [G(0) denotes fasting glucose] [16]. There are also methods based an assessment of glucose and insulin during a $75 \mathrm{~g}$ glucose tolerance test, such as the insulin resistance index (IRI), originally described by Belfiore et al. [17]. These methods involve an assessment of a dynamic change of glucose and insulin concentrations rather than during a steady (fasting) state. It is, however, not clear whether there is concordance of diagnosis of insulin resistance according to these methods.

The aim of the study was to compare prevalence of insulin resistance in a cohort of women with PCOS by means of the HOMA-IR and IRI methods.

\section{Material and methods}

The study involved 137 patients hospitalised in the Department of Endocrinology and Metabolic
Diseases of the Medical University of Lodz (Polish Mother's Memorial Hospital - Research Institute in 2013), i.e. all patients in whom a diagnosis of PCOS was unequivocally established during this year, according to the Rotterdam criteria (2003) [4]. The average age of the patients was (mean \pm SD) $25 \pm 7$ years, BMI $27.61 \pm 7.43 \mathrm{~kg} / \mathrm{m}^{2}$. Demographic as well as baseline metabolic characteristics of investigated patients are presented in Table I.

Insulin resistance index was calculated from changes of glycaemia and insulinaemia during a $75 \mathrm{~g}$ oral glucose tolerance test (OGTT) according to the method described by Belfiore et al. [17]. The IRI was calculated through the formula: ISI $\left.\right|_{(G \mid y)}$ $=2 /[1 /($ INSp $\times$ GLYp) $]+1$, where INSp and GLYp are the measured insulin and glycaemic areas. In normal subjects ISI(gly) is always around 1, with maximal variations between 0 and 2 . This method is based on changes of glycaemia and insulinaemia during OGTT, and correlates well with the euglycaemic hyperinsulinaemic glucose clamp technique [18]. According to some authors the assessment of free fatty acids (FFA) during OGTT is equally effective for the purpose of calculation of the IRI [17]. The cut-off point for this method is quoted as > 1.27 [19].

HOMA-IR index was calculated according to the formula: HOMA-IR = [glucose] $(\mathrm{mmol} / \mathrm{l}) \times$ [insulin $]$ $(\mu \mathrm{U} / \mathrm{ml}) / 22.5[15]$.

As there is no universal agreement as to the best cut-off for the HOMA-IR model, we adopted the most commonly used cut-off of 3.8 [20]. There are, however, data based on the analysis of the same, i.e. Spanish population, suggestive that a lower cut-off point (3.46) might be more appropriate for the $90^{\text {th }}$ percentile [21]. Hence, the testing was performed for both the abovementioned cut-off points for HOMA-IR model. The data were subsequently analysed by standard descriptive statistics and by both univariate (Spearman rank correlation) and multivariate models.

\section{Statistical analysis}

The Statistica 9.1 program was used for relevant calculations. Statistical significance was assumed for $p<0.05$.

\section{Results}

Mean HOMA-IR value was $2.72 \pm 2.24$ (median: 2.14, range: $0.33-16.78$ ). The prevalence of insulin resistance in the researched group was $49.6 \%$ (68/137) according to IRI, 22.6\% (31/137) and $15.8 \%(21 / 137)$ according to HOMA-IR (for the cutoff points of 3.46 and 3.8 , respectively). In cases of insulin resistance according to $\mathrm{IRI}_{1.27}$ there was concordance with $\mathrm{HOMA}-\mathrm{IR}_{3.46}$ in $83.9 \%$ of cases $(26 / 31)$, while in the case of HOMA-IR ${ }_{3.80}$, concor- 
Table I. Descriptive statistics for demographic and clinical data of examined group of 137 patients

\begin{tabular}{|c|c|c|c|c|c|c|}
\hline Parameter & Mean & $95 \% \mathrm{Cl}$ & Median & Min. & Max. & St. dev. \\
\hline Age & 25 & $23-26$ & 23 & 14 & 44 & 7 \\
\hline $\mathrm{BMI}\left[\mathrm{kg} / \mathrm{m}^{2}\right]$ & 27.61 & $26.39-28.83$ & 26.00 & 16.50 & 49.60 & 7.43 \\
\hline OGTT [mg/dl at $\left.120^{\prime}\right]$ & 102 & $98-106$ & 100 & 42 & 182 & 26 \\
\hline IRI (Belfiore) & 1.19 & $1.13-1.25$ & 1.24 & 0.28 & 1.86 & 0.36 \\
\hline Fasting glucose $[\mathrm{mg} / \mathrm{dl}]$ & 81 & $80-82$ & 80 & 59 & 102 & 7 \\
\hline Fasting insulin [mIU/ml] & 13.35 & $11.62-15.09$ & 10.49 & 2.25 & 87.14 & 10.60 \\
\hline HOMA-IR & 2.72 & $2.36-3.09$ & 2.14 & 0.33 & 16.78 & 2.24 \\
\hline $\mathrm{TSH}[\mathrm{mlU} / \mathrm{l}]$ & 2.05 & $1.89-2.21$ & 1.98 & 0.02 & 5.57 & 1.00 \\
\hline Free T4 [ng/dl] & 1.25 & $1.19-1.30$ & 1.20 & 0.80 & 4.21 & 0.35 \\
\hline HDL cholesterol [mg/dl] & 53 & $50-56$ & 51 & 29 & 206 & 18 \\
\hline LDL cholesterol [mg/dl] & 102 & $97-107$ & 100 & 38 & 191 & 29 \\
\hline Triglycerides [mg/dl] & 109 & $100-119$ & 102 & 30 & 265 & 55 \\
\hline Total cholesterol [mg/dl] & 165 & $160-171$ & 165 & 71 & 273 & 34 \\
\hline Oestradiol [pg/ml] & 77.4 & $62.3-92.4$ & 47.9 & 10.5 & 601.8 & 89.6 \\
\hline Total testosterone $[\mathrm{ng} / \mathrm{ml}]$ & 0.52 & $0.49-0.56$ & 0.50 & 0.14 & 1.2 & 0.20 \\
\hline DHEA-S $[\mu \mathrm{g} / \mathrm{dl}]$ & 310.40 & $\begin{array}{c}288.37- \\
332.43\end{array}$ & 284.10 & 54.80 & 936.9 & 133.28 \\
\hline Androstenedione [ng/ml] & 4.44 & $4.05-4.84$ & 3.92 & 1.19 & 12.4 & 2.35 \\
\hline
\end{tabular}

dance was noted in $85.7 \%$ of cases (18/21). On the other hand, the majority of patients found to be insulin-resistant according to IRI (> 1.27) were not insulin resistant according to HOMA-IR (42/68 $=61.7 \%$ and $50 / 68=73.5 \%$, for $\mathrm{HOMA}^{-\mathrm{IR}_{3.46}}$ and HOMA-IR ${ }_{3.80}$, respectively). There were only five and three cases of IR according to HOMA-IR with $\mid \mathrm{RI}<1.27$ (HOMA-IR $\mathrm{R}_{3.46}$ and HOMA-IR $\mathrm{R}_{3.80}$, respectively). Results of this analysis are presented in Tables II and III.

Interestingly, however, among the patients found to be insulin-resistant according to the IRI, those with a concomitant high HOMA-IR index were also found to have higher insulin resistance (Belfiore) indices ( $1.55 \pm 0.18$ vs. $1.44 \pm 0.14$, $p=0.014$, and $1.60 \pm 0.18$ vs. $1.44 \pm 0.13, p=$ 0.0008 , for HOMA-IR ${ }_{3.46}$ and HOMA-IR ${ }_{3.80}$, respectively). Hence, patients with high HOMA-IR (both $\mathrm{HOMA}-\mathrm{IR}_{3.46}$ and $\mathrm{HOMA}-\mathrm{R}_{3.80}$ ), generally tended to be more insulin resistant, with both methods applied.

In the next step we assessed the correlation between IRI and HOMA-IR models. The correlation between IRI and HOMA-IR was assessed both in the linear (Figure $1 \mathrm{~A}$ ) and non-linear models (Figure 1 B). There was a highly significant $(p<0.0001)$ but only moderate correlation between both models $(r=0.5$ and $r=0.57$ for a linear and non-linear model, respectively). Furthermore, in this model only $25 \%$ of the total variation in HOMA-IR can be explained by the relationship between HOMA-IR and IRI $\left(R^{2}=0.25, p<0.0001\right)$.

\section{Discussion}

The issue of insulin resistance in PCOS, though seemingly obvious, is indeed highly problematic, when supposed to be transformed from a theoretical concept into a clinical application. In a seminal paper by Dunaif et al. [22] insulin resistance in PCOS was assessed by means of euglycaemic glucose-clamp technique in obese $(n=19)$ and non-obese women with PCOS $(n=10)$ versus obese $(n=11)$ and non-obese controls $(n=8)$. The authors concluded that insulin resistance was apparent not in terms of exceeding a predefined cut-off point, but as decreased insulin sensitivity in comparison to BMI-matched non-PCOS peers (expressed as per kilogram total weight or per kilogram fat-free mass or when divided by the steady-state plasma insulin during a euglycaemic clamp). Hence application of any surrogate insulin resistance indices must be viewed with extreme caution.

Furthermore, it must be noted that there is no universal agreement as to the best cut-off point for various insulin-resistance indices. First of all, any cut-off points should be related to particular studied population, as significant ethnic differences have been reported [12]. Secondly, some 
Table II. Comparison of HOMA-IR and insulin resistance (Belfiore) indices for assessment of insulin resistance in women with polycystic ovary syndrome (cut-off for HOMA-IR > 3.46)

\begin{tabular}{|c|c|c|c|}
\hline \multirow[t]{2}{*}{$\mathrm{IRI}_{1.27}$} & \multicolumn{3}{|c|}{ HOMA-IR ${ }_{3.46}$} \\
\hline & $\leq 3.46$ & $>3.46$ & Total \\
\hline$\leq 1.27$ & $\begin{array}{c}64 \\
64 \times 100 \% / 69=92.75 \% \mathrm{HOMA} / \mathrm{IRI} \\
\text { concordance for subjects with IRI within } \\
\text { the reference range }\end{array}$ & $\begin{array}{c}5 \\
5 \times 100 \% / 69=7.25 \% \mathrm{HOMA} / \mathrm{IRI} \\
\text { discordance for subjects with IRI within } \\
\text { the reference range }\end{array}$ & 69 (50.4\%) \\
\hline$>1.27$ & $\begin{array}{c}42 \\
42 \times 100 \% / 68=61.8 \% \text { HOMA/IRI } \\
\text { discordance for subjects with raised IRI }\end{array}$ & $\begin{array}{c}26 \\
26 \times 100 \% / 68=38.2 \% \\
\text { HOMA/IRI concordance for subjects with } \\
\text { raised IRI }\end{array}$ & 68 (49.6\%) \\
\hline Total & $\begin{array}{c}106 \\
106 \times 100 \% / 137=77.4 \% \\
\text { subjects with HOMA-IR } \leq 3.46\end{array}$ & $\begin{array}{c}31 \\
31 \times 100 \% / 137=22.6 \% \\
\text { subjects with HOMA-IR > 3.46 }\end{array}$ & 137 \\
\hline
\end{tabular}

$P<0.0001$ (McNemar's test).

Table III. Comparison of HOMA-IR and insulin resistance (Belfiore) indices for assessment of insulin resistance in women with polycystic ovary syndrome (cut-off for HOMA-IR>3.80)

\begin{tabular}{|c|c|c|c|}
\hline \multirow[t]{2}{*}{$\mathrm{IRI}_{1.27}$} & \multicolumn{3}{|c|}{ HOMA-IR ${ }_{3.80}$} \\
\hline & $\leq 3.80$ & $>3.80$ & Total \\
\hline$\leq 1.27$ & $\begin{array}{l}66 \\
66 \times 100 \% / 69=95.7 \% \\
\text { HOMA/IRI concordance for subjects with IRI } \\
\text { within the reference range }\end{array}$ & $\begin{array}{c}3 \\
3 \times 100 \% / 69=4.3 \% \\
\text { HOMA/IRI discordance for subjects with IRI } \\
\text { within the reference range }\end{array}$ & $69(50.4 \%)$ \\
\hline$>1.27$ & $\begin{array}{c}50 \\
50 \times 100 \% / 68=73.5 \% \\
\text { HOMA/IRI discordance for subjects with } \\
\text { raised IRI }\end{array}$ & $\begin{array}{c}18 \\
18 \times 100 \% / 68=26.5 \% \\
\text { HOMA/IRI concordance for subjects with } \\
\text { raised IRI }\end{array}$ & $68(49.6 \%)$ \\
\hline Total & $\begin{array}{c}116 \\
116 \times 100 \% / 137=84.7 \% \\
\text { subjects with HOMA-IR } \leq 3.80\end{array}$ & $\begin{array}{c}21 \\
21 \times 100 \% / 137=15.3 \% \\
\text { subjects with HOMA-IR }>3.80\end{array}$ & 137 \\
\hline
\end{tabular}

$P<0.0001$ (McNemar's test).

authors use the $90^{\text {th }}$ percentile to define insulin resistance, for a particular (e.g. HOMA-IR) method $[20,21]$, while others suggest application of the $75^{\text {th }}$ percentile $[19,23,24]$. As a result of this, significantly lower cut-off points for HOMA-IR have been suggested, e.g. 2.5 [19], or 2.29 [23], or even 2.1 for the population of Krakow (Poland) [24]. Hence, arbitrary application of a pre-defined cutoff point without clear reference to characteristics of a particular studied population, and in the absence of genuine normative data of this population, is indeed problematic, and clearly cannot be validated as scientifically sound. The lack of standardised reference values among surrogate methods of assessment of insulin resistance has been recently raised by some authors [25].

The principal finding of our study is, however, a relatively weak association between the HOMA index derived from fasting glucose and insulin values and a Belfiore index, i.e. one of the methods based on assessment of glucose and insulin concentrations during OGTT. As this method, as well as its variations as described by Matsuda and DeFronzo [18], is principally based on assessment of the area under the curve for glucose and insulin excursions, such a relatively weak correlation $(r=0.5)$ would also apply to the insulin sensitivity index (ISI)/Matsuda index. As a result of this assessment of insulin resistance by the HOMA-IR index and IRI yields significantly different results according to the method applied. For instance, for a HOMA-IR cut-off point of 3.46, for 68 subjects with raised IRI, only 26 (38.2\%) had raised HOMA-IR. This difference was even more striking for a HOMA-IR cut-off of 3.8. The opposite situation, i.e. high HOMA-IR and "normal" IRI, was very uncommon and applied to only 5 (7.25\%) and $3(4.3 \%)$ subjects, for HOMA-IR cut-offs of 3.46 and 3.8, respectively. As a result, many more women with PCOS would be diagnosed as insulin resistant with IRI than with HOMA-IR, though those with high HOMA-IR generally tend to have higher IRI indices. This implies that the HOMA-IR index (for a $90^{\text {th }}$ percentile cut-off) also identifies the most insulin-resistant population according to the IRI method.

The discrepancy between HOMA-IR and IRI methods and their relatively weak correlation is 
A

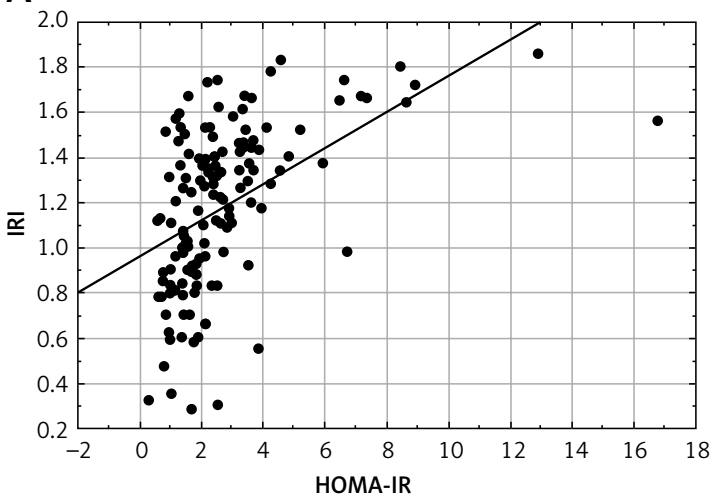

B

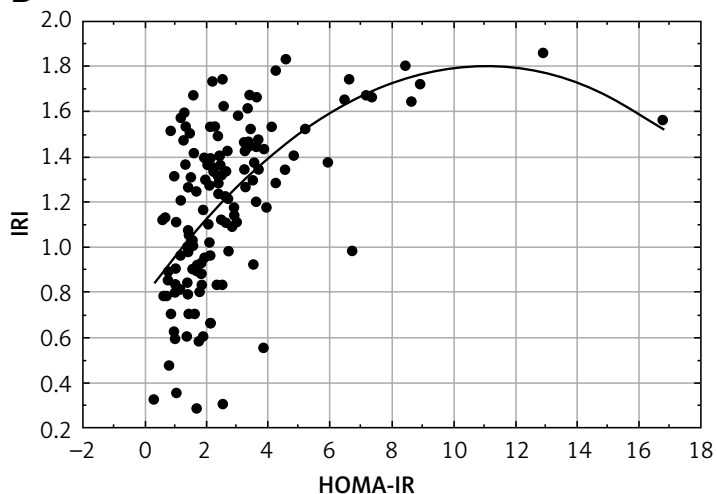

Figure 1. A - Correlation between IRI and HOMA-IR indices linear model $(r=0.50, p<0.001, n=137)$. B - Correlation between IRI and HOMA-IR non-linear model $(r=0.57, p<0.001, n=137)$

not surprising, given that insulin resistance indices derived from fasting glucose and insulin predominantly reflect hepatic rather than peripheral insulin sensitivity [26]. Furthermore, some studies have cast some doubt on the previously assumed excellent correlation of data obtained from these indices and data obtained from a euglycaemic clamp technique, both for fasting glucose and insulin models [27] and for methods based on glucose and insulin during OGTT [28]. In the latter case, some authors raise the issue that indices derived from OGTT could be subjected to many confounders [29]. For instance, significant reductions in $\beta$-cell function (where changes in corresponding glucose levels are initially mild) might significantly overestimate insulin sensitivity, while variation in gastric emptying might account for approximately $35 \%$ of the variance in peak blood glucose concentrations after ingestion of oral glucose [30], and so this may also seriously alter results obtained from OGTT-based methods.

In conclusion, our study clearly demonstrated that assessment of insulin resistance in women with PCOS is highly method-dependent, and that in a significant percentage of the studied population women might be classified either as "insulin resistant" or "insulin sensitive", according to the chosen method. Also there is no agreement as to what cut-off points should be used for surrogate measures of insulin resistance. Despite this, some clinicians use surrogate measures of insulin resistance, for instance in order to determine indications for metformin treatment. As the issue of the current place of metformin treatment in PCOS remains debatable, application of surrogate indices of insulin resistance as the sole determinant for the use of insulin-sensitising agents in women with PCOS must be viewed with extreme caution.

\section{Acknowledgments}

This study was financially supported by the Medical University of Lodz, Poland (project No.
503/1-107-03/503-11-001-18). We acknowledge a meticulous and sophisticated effort of dr Malgorzata Bienkiewicz from the Department of $\mathrm{Nu}$ clear Medicine of the Medical University of Lodz, who performed statistical analysis of the presented data.

\section{Conflict of interest}

The authors declare no conflict of interest.

\section{References}

1. Azziz R, Woods KS, Reyna R, Key TJ, Knochenhauer ES, Yildiz BO. The prevalence and features of the polycystic ovary syndrome in an unselected population. J Clin Endocrinol Metab 2004; 89: 2745-9.

2. Welt CK, Carmina E. Clinical review: lifecycle of polycystic ovary syndrome (PCOS): from in utero to menopause. J Clin Endocrinol Metab 2013; 98: 4629-38.

3. Ozegowska K, Pawelczyk KL. Cardiovascular risk in patients with polycystic ovary syndrome. Pol J Gynecol 2015; 86: 840-8.

4. Rotterdam ESHRE/ASRM-Sponsored PCOS Consensus Workshop Group. Revised 2003 consensus on diagnostic criteria and long term health risks related to polycystic ovary syndrome (PCOS). Hum Reprod 2004; 19: 41-7.

5. Bajuk Studen K, Jensterle Sever M, Pfeifer M. Cardiovascular risk and subclinical cardiovascular disease in polycystic ovary syndrome. Front Horm Res 2013; 40: 64-82.

6. Hart R, Doherty DA. The potential implications of a PCOS diagnosis on a woman's long-term health using data linkage. J Clin Endocrinol Metab 2015; 100: 911-9.

7. Lewandowski KC, Cajdler-Luba A, Bieńkiewicz M, Lewiński A. Women with oligo-/amenorrhoea and polycystic ovaries have identical responses to GnRH stimulation regardless of their androgen status: comparison of the Rotterdam and Androgen Excess Society diagnostic criteria. Neuroendocrinol Lett 2011; 32: 847-56.

8. Prelevic GM, Wurzburger MI, Balint-Peric, L, Nesic, JS. Inhibitory effect of sandostatin on secretion of luteinising hormone and ovarian steroids in polycystic ovary syndrome. Lancet 1990; 336: 900-3.

9. Poretsky L, Cataldo NA, Rosenvaks Z, Giudice LA. The insulin-related ovarian regulatory system in health and disease. Endocr Rev 1999; 20: 535-82. 
10. DeUgarte CM, Bartolucci AA, Azziz R. Prevalence of insulin resistance in the polycystic ovary syndrome using the homeostasis model assessment. Fertil Steril 2005; 83: 1454-60.

11. Legro RS, Kunselman AR, Dodson WC, Dunaif A. Prevalence and predictors of risk for type 2 diabetes mellitus and impaired glucose tolerance in polycystic ovary syndrome: a prospective, controlled study in 254 affected women. J Clin Endocrinol Metab 1999; 84: 165-9.

12. Wijeyarte CN, Balen AH, Barth JH, Belchetz PE. Clinical manifestations and insulin resistance (IR) in polycystic ovary syndrome (PCOS) among South Asians and Caucasians: is there a difference? Clin Endocrinol (Oxf) 2002; 57: 343-50.

13. Legro RS, Arslanian SA, Ehrmann DA, et al. Diagnosis and treatment of polycystic ovary syndrome: an Endocrine Society clinical practice guideline. J Clin Endocrinol Metab 2013; 98: 4565-92.

14. DeFronzo RA, Tobin JD, Andres R. Glucose clamp technique: a method for quantifying insulin secretion and resistance. Am J Physiol 1979; 237: E214-23.

15. Matthews DR, Hosker JP, Rudenski AS, Naylor BA, Treacher DF, Turner RC. Homeostasis model assessment: insulin resistance and beta-cell function from fasting plasma glucose and insulin concentrations in man. Diabetologia 1985; 28: 412-9.

16. Katz A, Nambi SS, Mather K, et al. Quantitative insulin sensitivity check index: a simple, accurate method for assessing insulin sensitivity in humans. J Clin Endocrinol Metab 2000; 85: 2402-10.

17. Belfiore F, lannello S, Volpicelli G. Insulin sensitivity indices calculated from basal and OGTT-induced insulin, glucose, and FFA levels. Mol Genet Metab 1998; 63: 134-41.

18. Matsuda M, DeFronzo RA. Insulin sensitivity indices obtained from oral glucose tolerance testing: comparison with the euglycemic insulin clamp. Diabetes Care 1999; 22: $1462-70$

19. Gutch M, Kumar S, Razi SM, Gupta JJ, Gupta A. Assessment of insulin sensitivity/resistance. Indian J Endocrinol Metab 2015; 19: 160-4.

20. Martizez-Larrad MT, Corbaton Anchuelo A, Del Prado N, Ibarra Rueda JM, Gabriel R, Serrano-Rios M. Profile of individuals who are metabolically healthy obese using different definition criteria. A population-based analysis in the Spanish population. PLoS One 2014; 9: e106641.

21. Gayoso-Diz P, Otero-Gonzalez A, Rodriquez-Alvarez MX, et al. Insulin resistance (HOMA-IR) cut-off values and the metabolic syndrome in a general adult population: effect of gender and age: EPIRCE cross-sectional study. BMC Endocr Dis 2013; 13: 47.

22. Dunaif A, Segal KR, Futterweit W, Dobrjansky A. Profound peripheral insulin resistance, independent of obesity, in polycystic ovary syndrome. Diabetes 1989; 38: 1165-74.

23. Radikova Z, Koska J, Huckova M, et al. Insulin sensitivity indices: a proposal of cut-off points for simple identification of insulin-resistant subjects. Exp Clin Endocrinol Diabet 2006; 114: 249-56.

24. Szurkowska M, Szafraniec K, Gilis-Januszewska A, Szybiński Z, Huszno B. Insulin resistance indices in population-based study and their predictive value in definig metabolic syndrome. Przegl Epidemiol 2005; 59: 743-51.

25. Szosland K, Lewiński A. In quest for method of insulin resistance assessment in everyday clinical practice - insulin resistance indices. Diabetes Metab Syndr Clin Res Rev 2016; 10S: S120-5.
26. Hoffman RP. Indices of insulin action calculated from fasting glucose and insulin reflect hepatic, not peripheral, insulinsensitivity in African-American and Caucasian adolescents. Pediatr Diabetes 2008; 9: 57-61.

27. Muniyappa R, Irving BA, Unni US, et al. Limited predictive ability of surrogate indices of insulin sensitivity/ resistance in Asian-Indian men. Am J Physiol Endocrinol Metab 2010; 299: E1106-12.

28. Kanauchi M, Tsujimoto N, Hashimoto T. Validation of simple indices to assess insulin sensitivity based on the oral glucose tolerance test in the Japanese population. Diabetes Res Clin Pract 2002; 55: 229-35.

29. Hücking K, Watanabe RM, Stefanovski D, Bergman RN. OGTT-derived measures of insulin sensitivity are confounded by factors other than insulin sensitivity itself. Obesity (Silver Spring) 2008; 16: 1938-45.

30. Horowitz M, Edelbroek MA, Wishart JM, Straathof JW. Relationship between oral glucose tolerance and gastric emptying in normal healthy subjects. Diabetologia 1993; 36: 857-62. 